\title{
An Integrated Post-locking, Multi-versioning, and Transformation Scheme for Consistency Maintenance in Real-time Group Editors
}

\author{
Liyin Xue \\ Department of computing \\ Macquarie University \\ Sydney, NSW 2109, Australia \\ lyxue@ics.mq.edu.au
}

\author{
Kang Zhang \\ Department of Computer Science \\ University of Texas at Dallas \\ Richardson, TX 75083-0688, USA \\ kzhang@utdallas.edu
}

\author{
Chengzheng Sun \\ School of Computing and IT \\ Griffith University \\ Brisbane, QLD 4111, Australia \\ C.Sun@cit.gu.edu.au
}

\begin{abstract}
Intention preservation is an important aspect of consistency maintenance in distributed group editors. In this paper, we first introduce a classification framework for various kinds of intentions, then propose an integrated post-locking, multiversioning, and transformation scheme (LOVOT) for intention preservation. With this scheme, individual users' compatible intentions are preserved by operational transformation, individuals' conflicting intentions are preserved by multiversioning, and the preservation of a syntactically and semantically consistent group intention is facilitated by postlocking. An algorithm for preserving individual users' intentions is discussed in details. The integrated scheme is fully distributed, highly responsive, non-blocking, and capable of avoiding locking overhead.
\end{abstract}

\section{Keywords}

Operational transformation, multi-versioning, post-locking, consistency maintenance, collaborative editors, $C S C W$.

\section{Introduction}

Distributed real-time group editors allow a group of users to view and edit the same document at the same time from geographically dispersed sites connected by communication networks in general, the Internet in particular.

In a distributed environment with non-deterministic communication latency, a replicated architecture is usually adopted for the storage of a shared document in order to meet the requirement for high responsiveness. The major challenge of supporting collaborative editing is the management of multiple streams of concurrent activities so that document consistency can be maintained in the course of conflicts. Various concurrency control techniques and algorithms have been proposed to tackle this issue $[4,6,7,8,12,18]$.

Locking is a widely used concurrency control technique in either database systems or collaborative applications. Conflicts can be prevented by locking, but locking is undesirable because it interrupts the users in their work and causes unnecessary overhead, that is, a locking operation has to be generated before editing operations.

Due to the restrictiveness of locking approaches, an unconstrained approach called operational transformation was proposed for consistency maintenance in real-time group editors [5]. It has been extended by several research groups since then $[3,14,16,17,19]$. Both its strength and weakness lie in its capability of intention preservation.

Intention preservation is an important aspect of consistency maintenance in group editors. The intention of an operation is the execution effect that can be achieved by applying it on the document state from which it was generated. The intentions of a user are represented by the intentions of operations which the user issues. Concurrent executions of operations may lead to intention violation. In this paper, we propose an integrated postlocking, multi-versioning, and transformation scheme for intention preservation in distributed real-time group text editors.

The rest of the paper is organised as follows. In Section 2 we present the integrated post-LOcking, multi-Versioning, and Operational Transformation scheme (LOVOT) for group text editing. Then we briefly present some background information and definitions in previous work, and introduce graphical notations for operational transformation in Section 3. Section 4 introduces a block-based plain text document model, and discusses the relationship amongst operations based on it. Next, an algorithm for individual users' intentions preservation $\left(\boldsymbol{I}^{2} \boldsymbol{P}\right)$ is discussed in Section 5. The integrated scheme is compared to related work in Section 6. Finally, major results are summarised and further work is discussed in Section 7.

\section{An integrated post-locking, multi- versioning, and transformation scheme}

In this section, we first examine the intention violation problems in transformation-based concurrency control approach, then identify the complementary roles of operational transformation, multi-versioning, and post-locking approaches to intention preservation in cooperative group editors. 


\subsection{Preserving individuals' compatible intentions by operational transformation}

A group editor must not only provide a convergent document state at each site, but also preserve each user's intention. Intention violation problems have been examined by groupware researchers recently $[2,12,15,19,20,21]$. For the presentation of this paper, we propose a classification framework for various kinds of intentions.

\subsubsection{Lower level intention and higher level intention}

A user's specific intention can be interpreted in different levels, i.e. basic level, syntactic level, and semantic level. Operational transformation approach can ensure plain strings be inserted/deleted at proper positions (i.e. maintaining basic level consistency) by transforming the parameters of operations. However, It fails in maintaining syntactic and semantic consistency.

For example, suppose a shared document contains a sentence

A: "John forgot lock the door."

There is an English grammar error in it. Assume user $i$ issues an operation $O_{1}$ to insert "to" at the position between "forgot" and "lock", thus changes sentence A to B. User $j$ concurrently issues an operation $\mathrm{O}_{2}$ to insert "ing" at the ending position of "lock", thus changes A to $\mathrm{C}$.

B: "John forgot to lock the door."

C: "John forgot locking the door."

The existing operational transformation schemes can preserve the basic level intentions of the two operations such that the final document contains sentence $\mathrm{D}$.

D: "John forgot to locking the door."

Apparently this result is still syntactically (or grammatically) incorrect, though both B and C are syntactically correct. Neither of the users' higher-level intentions is preserved. In addition, B and $\mathrm{C}$ have different meanings. It is not possible for the system to accommodate semantically conflicting intentions into one correct sentence.

\subsubsection{Compatible intention and conflicting intention}

If two operations target syntactically and semantically unrelated regions of a document, then operational transformation approach alone will preserve both of the users' intentions. We say that the two operations are transformable (or spatially compatible, see Section 4 for a formal definition). Otherwise, we say the two operations are non-transformable (or spatially conflicting). Obviously, the intentions of two transformable operations are compatible in the sense that both operations (properly transformed) can be applied to the document without interfering with each other. The intentions of two nontransformable operations are conflicting if they are concurrent. If operation $O_{1}$ causally precedes operation $O_{2}$, then their intentions should be considered as compatible, even though they are non-transformable (e.g.: Insert and Delete the same word).

\subsubsection{Individuals' intentions and group intentions}

An individual user's intention is represented by the intentions of one or more operations generated by a user. Thus individuals' intentions are a set of intentions of operations generated by a group of individual users. The intention of one user may conflict with some other users' intentions. A group intention represents the one which is agreed upon among the group. In other words, a group intention is the one resulting from merging individuals' intentions via a coordination or negotiation process among the involved users.

From the above discussion, we can conclude that the operational transformation approach is able to preserve basic level and compatible intentions of operations. Other techniques are needed to maintain syntactic and semantic consistency, or to preserve individuals' conflicting intentions and a group intention.

\subsection{Preserving individuals' conflicting intentions by multi-versioning}

A multi-versioning approach was proposed to preserve conflicting intentions (in our framework they are individuals' intentions) in cooperative graphics editors, such as GRACE and Tivoli $[2,11]$. When two concurrent operations change the same attribute of the same object to different values, intention violation occurs. It is impossible for the system to accommodate conflicting intentions in the same target object. The multiversioning approach preserves the intentions of both operations by making a new version from the original object, and then applying the two conflicting operations (i.e. ones with conflicting intentions) to the two versions separately. The resulting effect is that no conflicting operations are applied to the same version. Two compatible operations (i.e. ones with compatible intentions) can be applied to the same version without causing intention violation. In a highly concurrent real-time collaborative editing environment, a group of operations may have rather arbitrary and complex compatible and conflict relationships among them, their combined effect is a collection of versions. Each version is the result of the execution of a set of compatible operations. Therefore, the multi-versioning approach can accommodate individuals' conflicting intentions in a consistent way.

However the fine-grained multi-versioning approach proposed in GRACE and Tivoli also fails in preserving higher level intentions. For instance, in GRACE, one attribute of an object may syntactically or semantically depend on another attribute of the same object. Concurrent editing of them may cause intention violations. Both GRACE and Tivoli do not deal with this problem. Obviously object granularity should be carefully considered in order to preserve higher level intentions.

When adapting the basic idea of the multi-versioning approach to text editors, we should consider two additional factors. (1) Unlike graphic objects, characters or strings are not independent of each other. (2) The created versions can change the status (i.e., positions) of other characters and strings in the document. Due to these factors, the multi-versioning technique becomes much more complicated in text editing environments. 
In this paper, we adopt a block-based document model, where a block can be a line, sentence, paragraph, etc. When two concurrent operations target the same block, it will be replicated to accommodate the conflicting intentions. A branch document is introduced for each block in conflicting to refrain the multiversioning effect from interfering with the main document. All the versions created for one block are presented in one branch document. The more blocks in conflicting, the more branch documents will be created. The operational transformation approach is integrated to deal with the problems caused by dependency of blocks.

\subsection{Preserving group intentions by post-locking}

Although the multi-versioning approach provides a mechanism to accommodate conflicting operations in a consistent way, conflict resolution is entirely left to the users. The users can further edit any versions. In an extreme, a large number of versions may be created. It will be difficult for the users to reach an agreed upon result, i.e., a group intention.

Post-locking schemes were proposed to restrict the number of versions created, to manage the created versions, to facilitate the resolution of conflicts, and to preserve a group intention in cooperative graphical editors[21].

All former locking schemes have two things in common. First, locking is conflict preventing. Second, a lock request is issued before an editing operation in order to get a lock, either explicitly by a user or implicitly by the system. They can be categorised as pre-locking. In post-locking schemes, the locking request is not necessary. Locking will automatically be enforced by the system, when two or more concurrent operations target the same object. In the meantime, the users will be notified of the conflict.

In pre-locking, objects are locked to prevent potential conflicts, whereas the objects are always available except real conflicts arise in post-locking. Therefore, post-locking is more optimistic than conventional optimistic locking. In addition, locking operations are executed only on the local document in an autonomous way, thus no propagation is needed, in post-locking.

In this paper we adopt an approach called systemic postlocking, where no user except the system (local application) owns the locks on all the versions created, and users are not allowed to further edit any one of the versions before the lock is synchronised.

When the first conflict occurs at a site, two versions of the targeted block will be created in a branch document. Then the system locks the original block and the versions in the branch document, and the user at that site is not allowed to generate any further operation targeting them. Due to communication delays, operations may arrive in different orders at different sites. Newly arrived operations may conflict with both or one of the conflicting operations, they should be applied to the current versions in a proper way according to the multi-versioning approach. Any new versions created are locked by the system. It will take a period of time, called lock synchronisation period, for each site to reach a stable set of versions, which are locked and globally consistent. The system's locking operation reduces the complexity caused by the multiple version creation process to a minimal level. The major disadvantage is that all users have to wait for the synchronisation of locking before they can start to resolve the conflicts. Fortunately, this is acceptable, since the users can still edit other blocks that are not locked during this period of time. When the branch document is synchronised, the system may invoke a voting procedure or choose a representative to resolve the conflicts. Human users can easily resolve any syntactic and semantic intention violations caused by concurrent editing in a controlled environment obtained by post-locking.

From the above discussion, we can conclude that post-locking can facilitate human users in preserving group intentions and maintaining syntactic and semantic consistency. The latter was not proposed in [21].

\section{Definitions and notations}

In this section, the basic concepts and notations adopted in the REDUCE system (and GOTO algorithm), which is based on the operational transformation approach, are briefly presented. For details, the reader is referred to $[18,19]$. In the meantime we introduce new graphical notations to illustrate REDUCE's operational transformation functions.

\subsection{Contextual relations of operations}

Concurrent operations need to be properly transformed such that the effect of some serialised execution of them is equivalent to overall execution effect of all the operations. Each operation is generated on a particular document state. The context of a document state is the sequence of operations executed on the initial document state to arrive at the current document state. Given an operation $O$, the definition context of $O$, denoted as $D C(O)$, is the context of the document state on which $O$ is defined; and the execution context of $O$, denoted as $E C(O)$, is the context of the document state on which $O$ is executed. If two contexts contain the same set of operations, which may be executed in different orders and in different forms, we say they are equivalent.

Definition 1 Context equivalent relation “ $\Leftrightarrow$ ": Given two operations $O_{a}$ and $O_{b}, O_{a}$ and $O_{b}$ are context equivalent, expressed as $O_{a} \Leftrightarrow O_{b}$, iff $D C\left(O_{a}\right) \equiv D C\left(O_{b}\right)$, where “”” means two contexts are equivalent.

Definition 2 Context preceding relation " $\Rightarrow$ ": Given two operations $O_{a}$ and $O_{b}, O_{a}$ is context preceding $O_{b}$, expressed as $O_{a} \Rightarrow O_{b}$, iff $D C\left(O_{b}\right) \equiv D C\left(O_{a}\right)+\left[O_{a}\right]$ (where "+" expresses the concatenation of two operation lists).

The intention of an operation can be preserved if its definition context matches its execution context, i.e., $D C(O) \equiv E C(O)$.

REDUCE uses two primitive transformation functions, i.e., Inclusion Transformation (IT) and Exclusion Transformation $(E T)$, to transform an operation such that it can be executed in a new context while preserving its intention. An $I T\left(O_{a}, O_{b}\right)$ 
function transforms an operation $O_{a}$ against a context equivalent operation $O_{b}$ in such a way that the impact of $O_{b}$ is effectively included, whereas an $\operatorname{ET}\left(O_{a}, O_{b}\right)$ function transforms $O_{a}$ against a context preceding operation $O_{b}$ in such a way that the impact of $O_{b}$ is effectively excluded. When two operations' targeting regions happen to overlap, it is not easy to define the transformation functions.

\subsection{Graphical notations for operational transformation}

Operational transformation can be represented as graph transformation such that its effect is visualised and easier to be understood. Basic notations are presented in Fig 1. Two context equivalent concurrent operations can be inclusion-transformed and then executed consecutively (Fig 2 (a)). Two consecutively executed operations can be transformed such that they are context equivalent (Fig 2(b)). An operation $O$ can be inclusion(or exclusion-) transformed against a list of operations $(O L)$, i.e., $L I T(O, O L)$ or $L E T(O, O L)$ (Fig 2(c), (d)). We extend the $L E T$ function such that a list of operations $\left(O L_{a}\right)$ can be exclusiontransformed against another list $\left(O L_{b}\right)$, i.e., $\operatorname{LET}\left(O L_{a}, O L_{b}\right)$, as in Fig 2 (e).

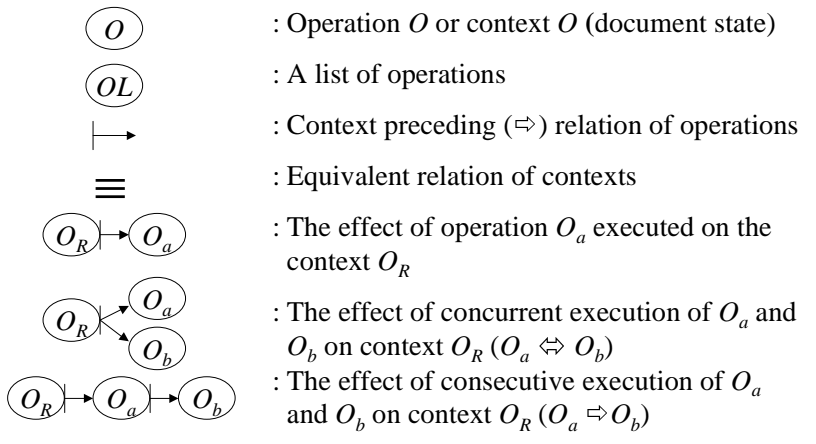

Fig 1 Basic notations of operational transformation

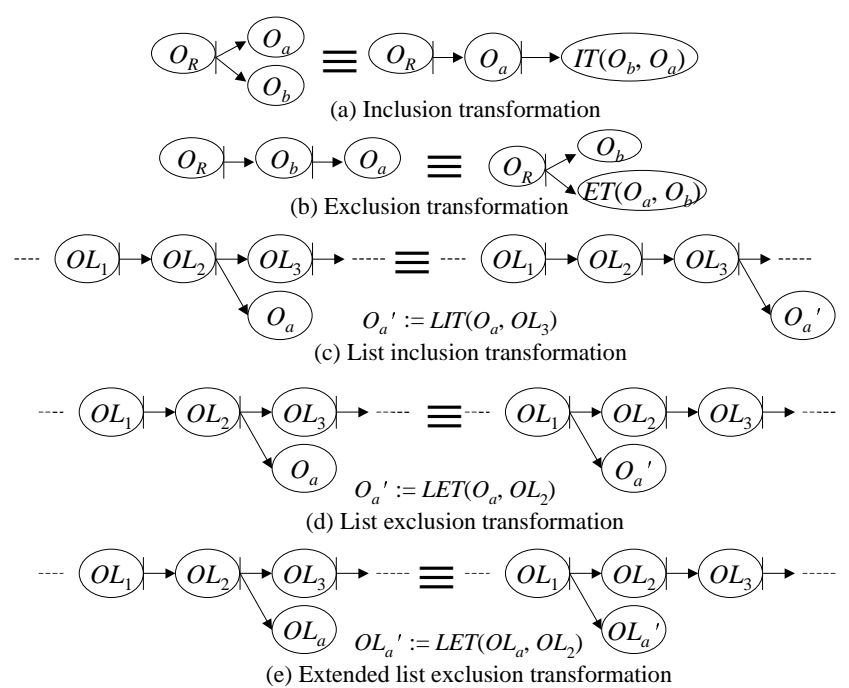

Fig 2 Transformation functions

\section{Document model and operation relationship}

\subsection{Block-based plain text document model}

We adopt a plain text document model in this paper, where a document is modelled as a linear sequence of lines, each of which contains a sequence of characters, addressed from 0 to the end of the document. This model does not maintain any character level attributes. A line represents a specific region in a document that begins from a starting position (after a newline character) and ends with a newline character.

In fact, the line in the above document model can be replaced with any unit of a document, e.g. sentence, paragraph, etc., each of which is identified by a delimiting symbol. Therefore, we use the term block for generality in this paper.

Generally speaking, blocks are semantically related in a document, otherwise they should not be put together. On the other hand, they are relatively independent, otherwise cooperative work would not make sense. We can assume any two blocks are syntactically and semantically independent as a first order approximation.

All replicated applications maintain a current state of the document that is being edited. For simplicity, we consider two primitive operations which modify the document's content without changing its structure, i.e. insert or delete a string which does not contain any delimiting symbols, e.g., newline characters $^{1}$. The two primitive operations are defined as follows:

Insert $[S, P, S P]$ : insert string $S$ at position $P$ of the block with a starting position of $S P$.

Delete $[N, P, S P]$ : delete $N$ characters started from position $P$ of the block with a starting position of $S P$.

Where $S P$ represents the absolute position of the block in the document, and $P$ is relatively addressed from $S P$.

\subsection{Spatial and intentional relations of operations}

Definition 3 Spatial conflict relation "ख్: Given two operations $O_{a}$ and $O_{b}$, they are spatially conflicting with each other, expressed as $O_{a}$ 凶 $O_{b}$, iff (1) $O_{a} \Leftrightarrow O_{b}$ and $\operatorname{SP}\left(O_{a}\right)=$ $S P\left(O_{b}\right)$, or (2) $O_{a} \Rightarrow O_{b}$ or $O_{b} \Rightarrow O_{a}$, and $S P\left(O_{a}\right)=S P\left(O_{b}\right)$, or (3) there exists an operation $O_{x}$, such that $O_{a} \bigotimes O_{x}$ and $O_{x} \bigotimes O_{b}$, where $\operatorname{SP}(O)$ denotes the starting position of operation $O$ 's target block.

Definition 4 Spatially compatible relation “ $\square$ ': Given two operations $O_{a}$ and $O_{b}$, if they do not spatially conflict with each other, they are spatially compatible, expressed as $O_{a} \square O_{b}$.

Definition 5 Intentional conflict relation “ $\otimes$ ": Given two operations $O_{a}$ and $O_{b}$, they are intentionally conflicting with each other, expressed as $O_{a} \otimes O_{b}$, iff (1) $O_{a} \| O_{b}$, and (2) $O_{a}$ 冈 $O_{b}$, where ""l" denotes the independent (or concurrent) relation of the operations. We will use " $\rightarrow$ " to denote the causal ordering relation in this paper.

\footnotetext{
1 For structure changing operations, new definition of spatial relation and transformation functions are needed, but the generic algorithm discussed in the coming sections remains unchanged.
} 
Definition 6 Intentionally compatible relation “○”: Given two operations $O_{a}$ and $O_{b}$, if they do not intentionally conflict with each other, they are intentionally compatible, expressed as $O_{a} \odot O_{b}$.

In the following sections, we use "conflict (compatible)" to represent "intentionally conflict (compatible)" for simplicity. It should be clear from the context.

\subsection{Transformable and non-transformable operation lists}

If two operations spatially conflict with each other, then it is pointless to transpose them (i.e., to change the execution order). Therefore, it is unnecessary to consider such a case in defining the transformation functions, and the design of them will become much easier.

Definition7 Transformable, semi-transformable and nontransformable operation lists: Given a list of intentionally compatible operations $O L$, for $\forall O_{i}, O_{j} \in O L(i \neq j)$, if $O_{i} \square O_{j}$, then the list is transformable; else if $O_{i} \llbracket O_{j}$, then the list is nontransformable; otherwise, the list is semi-transformable.

The operations in a transformable list can be executed in any order (after proper transformations) without changing the intentions. The operations in a non-transformable list can only be executed in one order specified by the list (in fact, they are a list of operations targeting the same block in the causal order). Any semi-transformable list can be transposed into a list consisting of several non-transformable sub-lists.

However, with the introduction of spatial conflict, list transformation functions, i.e., $\operatorname{LIT}(O, O L)$ and $\operatorname{LET}(O, O L)$ hold only if $O \square O L$, i.e., $O$ is spatially compatible with all the operations in $O L$. As for $\operatorname{LET}\left(O L_{a}, O L_{b}\right), O L_{a} \square O L_{b}$ must be satisfied (but two operations within a list may spatially conflict with each other) (The functions in detail are omitted).

\subsection{Inclusion and exclusion transformation functions}

To facilitate the description of transformation functions, in addition to the above $S P(O)$, we introduce the following notations: (1) Type $(O)$ : the type of operation $O$, i.e. Insert or Delete. (2) $P(O)$ : the position parameter of operation $O$. (3) $L(O)$ : the length parameter of operation $O$. For Insert, it is the length of the string to be inserted; for Delete, it is the number of characters to be deleted. (4) $S(O)$ : the string of the Insert or Delete operation. For the text editing application domain, we can define the inclusion and exclusion transformation functions as follows (We present them in one function to save space. They are separated by “"”).

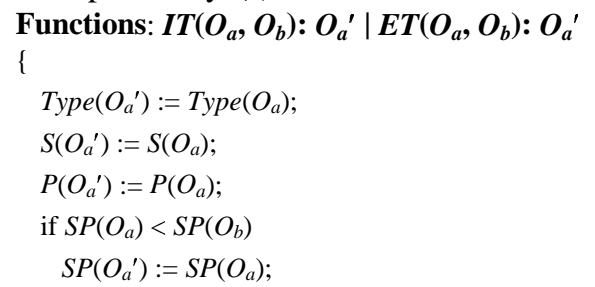

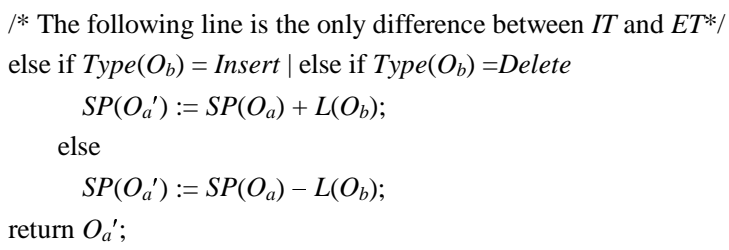

Due to the assumption that when two operations target the same block, i.e. $O_{a} \bowtie O_{b}$, no transformation is possible, both of the functions become very simple. It is natural that operation type and the string inserted or deleted remain unchanged after transformation. The position parameter $P(O)$ is relatively addressed, so it remains unchanged as well. The only parameter changed after transformation is $\operatorname{SP}(O)$.

\section{Algorithm for preserving Individuals' intentions}

In this section, we discuss the basic data structures and algorithms for preserving individuals' intentions.

\subsection{Interaction history of group editing}

A History Buffer $(H B)$ is needed to keep track of executed editing operations to support operation transformation. It maintains all global editing operations applied to the main document. In order to support multi-versioning process, we need to introduce a Conflict History Tree $(C H T)$ to record the set of operations concurrently targeting the same block and thus being applied to a branch document. When conflicts are resolved, the CHT will be garbage-collected. The major complexity of LOVOT scheme lies in how to update the $H B$ and $C H T$ s when executing an operation $\left(O_{\text {new }}\right)$ from a remote site.

When an operation is generated at a remote site, there may exist a number of $C H T$ s associated with its definition context. Each $C H T$ represents the state of a block currently in conflicting. A Locking Table $(L T)$ is introduced to keep track of all the conflicts happened in the whole document. Each entry in the $L T$ represents information about a locked block and its replicated versions, such as, current starting position $(S P)$ of the locked block, pointer to the corresponding $C H T$. As soon as the group intention is applied to the main document, the corresponding lock entry will be deleted from the table.

\subsubsection{Structure of history buffer}

Operations are executed according to their causal order, i.e., only causally ready operations can be executed without being suspended. The execution form of a newly arrived remote operation depends on its spatial and temporal relations with the operations in the local $H B$ and $C H T$ s.

Preceding and concurrent history lists $\left(H L_{P R}\right.$ and $\left.H L_{C C}\right)$ : We can transpose the $H B$ into two parts, i.e., preceding history list $\left(H L_{P R}\right)$ and concurrent history list $\left(H L_{C C}\right)$. All operations in $H L_{P R}$ causally precede $O_{\text {new }}$, denoted by $H L_{P R} \rightarrow O_{\text {new }}$; and $O_{\text {new }}$ 
is independent of all the operations in $H L_{C C}$, denoted by $O_{\text {new }} \|$ $H L_{C C}$.

Compatible and conflict history lists $\left(H L_{C P}\right.$ and $\left.H L_{C F}\right)$ : The concurrent history list can be further transposed into compatible history list $\left(H L_{C P}\right)$ and conflict history list $\left(H L_{C C}\right)$ in terms of spatial relations between its operations and $O_{\text {new. }}$. All operations in $H L_{C P}$ are compatible with $O_{\text {new }}$, denoted by $H L_{C P}$ $\odot O_{\text {new }}$; and $O_{\text {new }}$ conflicts with all the operations in $H L_{C F}$, denoted by $O_{\text {new }} \otimes H L_{C F}$. $H L_{C F}$ is a non-transformable operation list.

\subsubsection{Structure of conflict history tree}

All operations targeting the same block during an editing session can be represented as a directed tree (Fig 3), where a node denotes an operation, an arc denotes the execution order (and also the causal order, which is different from the arc in Fig 1 , where two operations connected may have been generated concurrently) of the two operations connected. An operation translates the block from one state to another. Any branching indicates there were concurrent editing operations from various sites. All the operations within the dashed box involved in conflicts. We can remove this part from the snapshot, and append it under a dummy root node $\left(O_{R}\right)$, which represents the context of the operations directly under it (e.g., $\mathrm{O}_{1}, \mathrm{O}_{2}$ ), thus we get a Conflict History Tree (Fig 4). We transform the CHTs (or the starting positions: $S P(O))$ such that they are defined on the current main document state, i.e., all the dummy root nodes of current $C H T$ s represent the same local history in the main document. Thus we say that all the $C H T$ s are context equivalent.

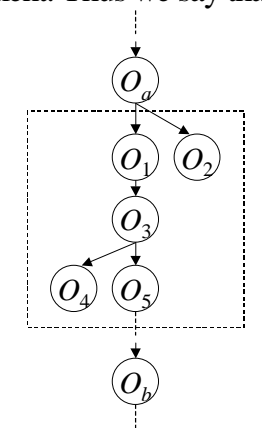

Fig 3 A snapshot of editing history in a block

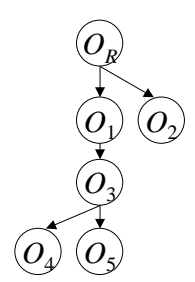

Fig 4 An example of $C H T$
Dynamically, a $C H T$ is created as long as a remote operation $O_{\text {new }}$ conflicts with one or more operations (i.e., $H L_{C F}$ ) in the local history buffer. The initial $C H T$ contains only two branches, i.e., $O_{\text {new }}$ and $H L_{C F}$, which is removed from the $H B$ when the conflict occurs. If a later received remote operation is targeting the same block in conflicting, then it will be directly inserted into the tree, without being recorded in the local $H B$. Operations involved in the conflicts are applied to the tree according to their temporal relationships (they are spatially conflicting anyway). No further operations targeting the block are allowed to be generated when the conflict has been detected, i.e., the block is locked. Therefore if a site has the knowledge that the first couple of conflicting operations detected have been executed at all the participating sites, it can conclude that the lock is synchronised and the versions become stable [21]. A synchronised $C H T$ represents a branch document consisting of a collection of globally consistent versions, each of which is represented by the operations in a path from the root to a leaf of the $C H T$. The operation identifiers of the stable leaf nodes can be used to identify the corresponding versions.

For instance, in Fig 4 there are three stable versions (three paths from the root to each leaf node), represented by three version history lists: $\left[\mathrm{O}_{2}\right],\left[\mathrm{O}_{1}, \mathrm{O}_{3}, \mathrm{O}_{4}\right]$, and $\left[\mathrm{O}_{1}, \mathrm{O}_{3}, \mathrm{O}_{5}\right]$. Operations are causally ordered within a list.

When $O_{\text {new }}$ is ready to execute at the local site, the local context may be different from the remote one. Some operations concurrent with $O_{\text {new }}$ may have been applied to the main document (and inserted into local $H B$ ), while others may conflict with some of the operations in $O_{\text {new }}$ 's definition context and cause a removal of them from the local $H B$ to $C H T$ s . Therefore, the local $H L_{P R}$ may contain fewer operations than $O_{\text {new }}$ 's definition context $H B$.

An algorithm can be easily devised to collect all those operations that are in the local CHT (denotes all the current $C H T$ s in the local site), but were in the remote $H B$ when $O_{\text {new }}$ was generated. We call them HBtoCHT operations for convenience. Each $\mathrm{CHT}$ may have zero, one or more HBtoCHT operations, but can be represented by an equivalent composite operation (ECO) (The technical details are omitted). If a $C H T$ has no HBtoCHT operation, then it is represented by an identity operation. As all the $C H T$ s are context equivalent (equivalent root nodes), the ECOs can be list-inclusion-transformed to get a final equivalent list, i.e., $E L$, which consists of $|\mathbf{C H T}|$ operations (|CHT| denotes the number of CHTs in the local site). It is a transformable operation list, and $H B[|H B|] \Rightarrow E L[1], E L[i] \Rightarrow E L[i$ $+1](i=1, \ldots,|\mathbf{C H T}|-1)$.

\subsection{Algorithm for preserving individuals' intentions}

A remote operation $O_{\text {new }}$ may be applied to either the main document or a branch one. In order to preserve its intention, we first need to reconstruct its definition context $\left(D C\left(O_{\text {new }}\right)\right)$ at local site, and then exclusion-transform it against those concurrent operations, such that it can be executed in the current local context $\left(E C\left(O_{\text {new }}\right)\right)$. The major challenges are how to get the decomposed history lists, and how to determine the spatial relation between two operations that may have an arbitrary contextual relation.

In the $\boldsymbol{G O T O}$ algorithm [18], since $O_{\text {new }}$ 's definition context is equivalent to $H L_{P R}$, and $O_{n e w}$ is context equivalent to $H L_{C C}[1]$, its execution form $\left(E O_{\text {new }}\right)$ can be obtained simply by list-exclusiontransforming it against $H L_{C C}$, as illustrated in Fig 5. However, with the introduction of the multi-versioning approach, the intention preservation procedure becomes much more complicated. 


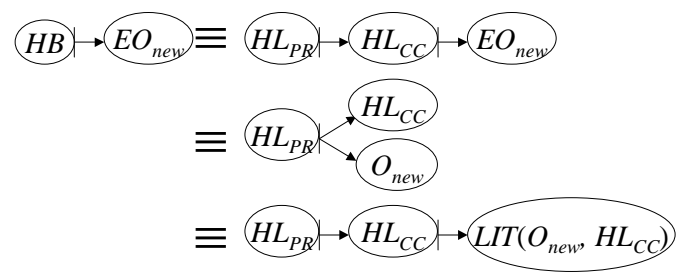

Therefore $E O_{\text {new }}=\operatorname{LIT}\left(O_{\text {new }}, H L_{C C}\right)$

Fig 5 Operational transformations in GOTO algorithm

$$
\underset{T E L:=L E T\left(E L, H L_{C C}\right) \quad\left(\text { since } E L \square H L_{C C}\right)}{H} \rightarrow H L_{C C} \rightarrow E L
$$

(a) Reconstructing $O_{\text {new }}$ 's definition context

$$
\begin{aligned}
& \text { If } O_{\text {new }} \text { घTEL, then } T O_{\text {new }}:=L E T\left(O_{\text {new }}, T E L\right) \\
& \text { Otherwise, } O_{\text {new }} \text { conflicts with one of the } C H T \mathrm{~s} \\
& \text { (b)Transforming } O_{\text {new }} \text { such that } T O_{\text {new }} \Leftrightarrow H L_{C C}[1]
\end{aligned}
$$

(c) Obtaining $O_{\text {new }}$ 's execution form at the local site

Fig 6 Operational transformations in $I^{2} P$ algorithm

From the previous discussion, it is clear that $O_{\text {new }}$ 's definition context contains $H L_{P R}$ and $H B t o C H T$ operations. $H L_{P R}$ can be obtained by transposing the local $H B$ into two consecutive operation lists, i.e., $H L_{P R}+H L_{C C}$ as in the GOTO algorithm. Since the definition context of $E L$ is the $H B$, i.e., $H B[|H B|] \Rightarrow$ $E L[1]$, it needs to be list-exclusion-transformed against $H L_{C C}$ such that $H L_{P R}\left[\left|H L_{P R}\right|\right] \Rightarrow T E L[1]$, and $H L_{C C}[1] \Leftrightarrow T E L[1]$, where $T E L=L E T\left(E L, H L_{C C}\right)^{1}$ (Fig 6(a)). Because of $E L \square$ $H L_{C C}$, the list exclusion transformation is possible.

Now that $D C\left(O_{\text {new }}\right)=H L_{P R}+T E L, O_{\text {new }}$ can be list-exclusiontransformed against $T E L$ such that $T O_{\text {new }}$ is context equivalent to $H L_{C C}[1]$, where $T O_{\text {new }}=\operatorname{LET}\left(O_{\text {new }}, T E L\right)($ Fig $6(\mathrm{~b}))$. Since $O_{\text {new }}$ may spatially conflict with one of the equivalent composite operations, the above list transformation does not apply. Moreover, it is not possible to detect spatial compatibility of two operations with an arbitrary contextual relation without proper transformations. Nevertheless, we can check the compatibility between $O_{\text {new }}$ and $T E L[|\mathbf{C H T}|]$ because the latter is context preceding the former. If they are not compatible, $O_{\text {new }}$ is spatially conflicting with the corresponding $C H T$ and should be appended to an appropriate branch of it, thus the execution of $O_{\text {new }}$ is done. Otherwise, $O_{\text {new }}$ can be exclusion-transformed against $T E L[|\mathbf{C H T}|]$ such that $T E L[|\mathbf{C H T}|-1] \Rightarrow T O_{\text {new }}$, where $T O_{\text {new }}=$

\footnotetext{
${ }^{1} T E L$ stands for Temporary $E L$ - a notation used to represent an intermediate result during transformation. Similarly $T O_{\text {new }}$ stands for Temporary $O_{\text {new }}$.
}

$E T\left(O_{\text {new }}, T E L[|\mathbf{C H T}|]\right)$. It is now possible to detect the compatibility of $T E L[|\mathbf{C H T}|-1]$ and $T O_{\text {new }}$. This one by one detection and transformation process can continue backward against the operation list until a conflict is detected or the entire list is exhausted. If $O_{\text {new }}$ is compatible with all the current local $C H T$ s, we obtain an intermediate $T O_{\text {new }}$ which is context equivalent to $H L_{C C}[1]$.

If $T O_{\text {new }} \square H L_{C C}$, then simply list-inclusion-transforming $T O_{\text {new }}$ against $H L_{C C}$ will get the execution form $E O_{\text {new }}$ in the local context. If $H L_{C F} \neq\left[\right.$ [], then it is not possible to transform $T O_{\text {new }}$ against any one of the operations in $H L_{C F}$. Fortunately it is possible to transpose $H L_{C C}$ into two consecutive operation lists, i.e. $H L_{C P}+H L_{C F}$, since $H L_{C P} \square H L_{C F}$. In the mean time, $T O_{\text {new }}$ can be inclusion-transformed against $H L_{C P}$ such that $E O_{\text {new }}$ and $H L_{C F}[1]$ are context equivalent, where $E O_{\text {new }}=\operatorname{LIT}\left(T O_{\text {new }}, H L_{C P}\right)$ (Fig 6 (c)). Similar to the exclusion-transformation of $O_{\text {new }}$ against $T E L$, the transformation of $T O_{\text {new }}$ against any operation in $H L_{C C}$ should be done one by one in order to detect their spatial compatibility. In the most general case, we obtain $E O_{\text {new }}$ 冈 $H L_{C F}, E O_{\text {new }} \| H L_{C F}$, and $E O_{\text {new }} \Leftrightarrow H L_{C F}[1]$. Noting that $S P\left(E O_{\text {new }}\right)=S P\left(H L_{C F}\left[H L_{C F}\right]\right)$, we can now determine the spatial relations between $E O_{\text {new }}$ and the $C H T$ s, and apply $E O_{\text {new }}$ to either the main document $(H B)$ or a branch one $(C H T)$. The possible results of executing a remote operation are as follows:

(1) If $\left(E O_{\text {new }} \square\right.$ - CHT $) \wedge\left(E O_{\text {new }} \square H L_{C C}\right.$, i.e. $\left.H L_{C F}=[]\right)$, then $E O_{\text {new }}$ is appended at the end of the local $H B$, the main document is updated according to the intention of $E O_{\text {new }}$. Any existing branch documents remain unchanged, though their starting positions $(S P)$ recorded in the locking table should be updated, i.e. inclusion-transformed against $E O_{\text {new }}$.

(2) If $\left(E O_{\text {new }} \square\right.$ CHT $) \wedge\left(E O_{\text {new }}\right.$ \ $H L_{C F}$, i.e. $H L_{C F} \neq[$ ] ] $)$, then a new $C H T$ is created with two branches under a root node, one is $H L_{C F}$, the other is $E O_{\text {new }}$. The $H L_{C F}$ is removed from the $H B$. The main document is updated by undoing the operations in $H L_{C F}$ in a reverse order (the reverse operations will not be stored in the $H B$ ). Any existing branch documents remain unchanged, but their starting positions need to be list-exclusion-transformed against the undone operations in $H L_{C F}$.

(3) If $\left(E O_{\text {new }} \otimes C H T_{i}, C H T_{i} \in \mathbf{C H T}\right) \wedge\left(E O_{\text {new }} \square H L_{C C}\right.$, i.e. $H L_{C F}=$ []), then $E O_{\text {new }}$ is inserted into the tree as a child of the root node, and a new version is created in the branch document. The main document and any other branch documents remain unchanged.

(4) If ( $E O_{\text {new }}$ ख $\left.C H T_{i}, C H T_{i} \in \mathbf{C H T}\right) \wedge\left(E O_{\text {new }}\right.$ ख $H L_{C F}$, i.e. $H L_{C F} \neq$ []), then the $C H T_{i}$ will be replaced by a combination of $H L_{C F}, E O_{\text {new }}$ and $C H T_{i}$, denoted by $<H L_{C F}, E O_{\text {new }}$, $C H T_{i}>$, in terms of their temporal and contextual relations. The $H L_{C F}$ is removed from the $H B$. The main document is updated by undoing the operations in $H L_{C F}$ in a reverse order. Any other branch documents remain unchanged, but their starting positions need to be list-exclusion-transformed against $H L_{C F}$.

(5) In addition, As pointed out before, $O_{\text {new }}$ may spatially conflict with one of the operations in the list $T E L$, i.e., it conflicts with a $C H T$ which has some operations causally preceding it. In this case, $O_{\text {new }}$ is appended at the end of the 
path composed of the operations preceding it. The main document and any other branch documents remain unchanged. The only difference between possible effects (3) and (5) is the position within a $C H T$, where $O_{\text {new }}$ is appended.

The possible execution effects are illustrated in Fig 7.

$$
H L_{P R} \rightarrow \rightarrow H L_{C P} \rightarrow \underset{E L_{\text {new }}}{H L_{C F}} \rightarrow \text { CHT }
$$

Denotes a contextual relation too, but the operations

following it are not applied to the main document.

(a) $E O_{\text {new }}$ 's definition context

$$
\begin{aligned}
& H B \rightarrow E O_{\text {new }} \rightarrow I T\left(\mathbf{C H T}, E O_{\text {new }}\right) \\
& H L_{C F}=\text { [], } E O_{\text {new }} \text { चCHT }
\end{aligned}
$$

(To transform a $C H T$ means to update its $S P$ in the locking table)

(b) $E O_{n e w}$ is applied to the main document (possible effect (1))

$$
\begin{aligned}
& H L_{P R} \rightarrow \rightarrow H L_{C P} \rightarrow \frac{L E T\left(\mathbf{C H T}, H L_{C F}\right)}{\left\langle E O_{\text {new }}, H L_{C F}\right\rangle} \\
& H L_{C F} \neq \text { [], } E O_{\text {new }} \text { चC } \mathbf{C H T}
\end{aligned}
$$

(A new $C H T$ consists of all the operations within the pair of brackets)

(c) A new branch document is created (possible effect (2))

$$
\begin{aligned}
& H L_{P R} \rightarrow \rightarrow H L_{C P}<\frac{L E T\left(\mathbf{C H T}^{\prime}, H L_{C F}\right)}{\left.<H L_{C F}, E O_{\text {new }}, C H T_{j}\right\rangle} \\
& E O_{\text {new }} \text { 冈 } C H T_{i}, E O_{\text {new }} \text { चCHT' }
\end{aligned}
$$

(CHT' denotes all CHTs except the one conflicting with $E O_{\text {new }}$, i.e., $C H T_{i}$.) (d) $E O_{\text {new }}$ is applied to an existing branch document

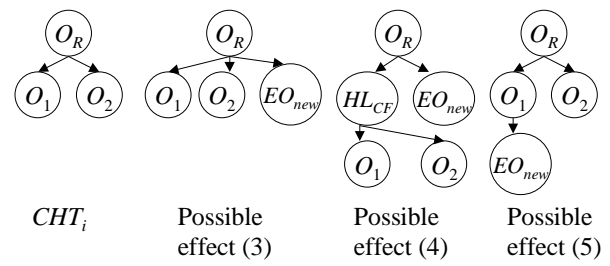

(e) Examples of an updated $C H T$ : $\left\langle H L_{C F}, E O_{n e w}, C H T_{i}\right\rangle$

Fig 7 Possible effects of executing $O_{\text {new }}$

The detailed algorithm for Individuals' Intentions Preservation $\left(\boldsymbol{I}^{2} \boldsymbol{P}\right)$ is omitted due to short of space.

As soon as the lock on a block is synchronised, the involved users will be notified. The conflicts can be resolved either by lock transferring or voting technique [21]. Further discussions of conflict resolution and group intention preservation are beyond the scope of this paper.

\section{Comparison to related work}

The operational transformation approach was pioneered by Ellis and Gibs in the GROVE system [5], and extended by a number of research groups around the world. Example systems and algorithms are: the REDUCE system (GOT algorithm) [19], the Jupiter system [14], the adOPTed [17], the CCU [3], and the GOTO [18] algorithms. The graphical notations presented in this paper visualise the operational transformation process in the GOTO algorithm. The extended algorithms can preserve basic level intentions of operations, but fail in preserving individual users' syntactic and semantic intentions when concurrent operations target the same region of a document. No mechanism was provided to support the resolution of higher level intention violations.

A variety of locking schemes have been proposed to maintain consistency in group text editors, such as the MACE [13], the SHREDIT [10], the SASSE [1], and the DISTEDIT [9] locking schemes. A sophisticated locking scheme with multiple granularity and compatibility is proposed in [12]. All the schemes can be categorised as pre-locking approaches. Although being able to preserve individual users' intentions applied to a locked block, pre-locking is too restrictive for highly responsive and highly concurrent distributed group editors. It introduces unnecessary locking overheads, and prevents other users from expressing their intentions on the same area. In contrast to prelocking, post-locking approaches allow conflicts to occur, and provide mechanisms to manage and resolve the conflicts in order to realise a group intention. Therefore the pre-locking overheads are eliminated completely and consequently the responsiveness characteristic of single user editors is preserved.

The multi-versioning approach proposed in Tivoli [11] and GRACE [2] fails in preserving syntactic and semantic consistency. They are based on an assumption that primitive graphic objects (e.g. line, circle, etc.) in a document are mutually independent. In this paper we extend the multi-versioning approach to a block-based document model, where blocks are independent of each other in syntactic and semantic level, but dependent in basic level (position of a block), thus operational transformation is needed. Our LOVOT scheme can resolve syntactic and semantic intention violations occurred within a block.

In fact, the basic multi-versioning strategy has also been used in cooperative text editors, when there are two concurrent Insert operations inserting two strings or characters at the same position, both of them are maintained in the document rather than being merged into one [17, 19]. Nevertheless it is not possible to accommodate other combinations of conflicting operations in such a simple way. We introduce branch documents, which are represented by a data structure called conflict history tree, to present the blocks in conflicting, such that all users' intentions targeting the same block can be accommodated in it in a globally consistent way. In addition, from the point of view of algorithm design, it reduces the problem of interfering with the main document $(H B)$, and thus facilitates the operational transformation processes.

Both Tivoli and GRACE rely on users manually resolving the conflicts without providing any further support except a conflict awareness mechanism. This paper adopts a post-locking approach to support users resolving the conflicts and reaching a consistent group intention. Although the approach is able to facilitate human users in preserving syntactic and semantic consistency, this functionality was not proposed in [21]. Moreover, the introduction of branch document also facilitates 
users' conflict awareness and resolution. Any versions created are mixed with the main document in Tivoli and GRACE, whereas all versions of the block in conflicting are rendered in a separate branch document in our approach.

Finally, most of the complexities of designing an application dependent transformation function come from dealing with the cases of spatially conflicting operations. For example, very complicated transformation functions were designed for stringwise primitive editing operations in REDUCE [19]. With the introduction of the multi-versioning approach, all the cases of conflicts are controlled by the generic individuals' intentions preservation $\left(\boldsymbol{I}^{2} \boldsymbol{P}\right)$ algorithm, thus application dependent transformation functions become much simpler.

\section{Conclusions and future work}

In this paper, we have presented a classification framework for various kinds of intentions, and identified the complementary roles of post-locking, multi-versioning, and operational transformation approaches in intention preservation. Based on them, an integrated scheme (LOVOT) has been proposed for consistency maintenance in distributed cooperative group text editors. With this scheme, individual users' compatible intentions are preserved by operational transformation, individuals' conflicting intentions are preserved by multiversioning, and the preservation of a syntactically and semantically consistent group intention is facilitated by postlocking. Technically, an algorithm for preserving individuals' intentions $\left(\boldsymbol{I}^{2} \boldsymbol{P}\right)$ has been discussed in details. The LOVOT scheme is fully distributed, highly responsive, non-blocking, and capable of avoiding locking overhead.

We are currently working on the formal specification and verification of the integrated scheme and algorithms, and extending them to more general document models. Work is underway to investigate group undo mechanisms within the framework of our integrated scheme.

\section{References}

[1] R. M. Baecher, et al. The user-centered iterative design of collaborative writing software. In ACM INTERCHI'93 Conference Proceedings, April 1993, pp.399--405.

[2] D. Chen and C. Sun. A distributed algorithm for graphic objects replication in real-time group editors. In Proc. of the International ACM SIGGROUP Conference on Supporting Group Work, Nov. 1999, pp.121--130.

[3] C. V. Cormack. A calculus for concurrent update. Research report CS-95-06, Dept. of Computer Science, University of Waterloo, Canada, 1995.

[4] P. Dourish. Consistency guarantees: exploiting application semantics for consistency management in a collaboration toolkit. In Proc. of the ACM Conference on CSCW, Nov. 1996, pp.268--277.

[5] C.A. Ellis and S.J. Gibbs. Concurrency control in groupware systems. In Proc. of ACM SIGMOD Conference on Management of Data, May 1989, pp.399--407.
[6] C.A. Ellis, S.J. Gibbs, and G.L. Rein. Groupware: some issues and experiences. In Communications of ACM 34 (1), Jan.1991, pp.39--58.

[7] S. Greenberg and D. Marwood. Real time groupware as a distributed system: concurrency control and its effect on the interface. In Proc. ACM Conference on CSCW, Nov. 1994, pp.207--217.

[8] A. Karsenty and M. Beaudouin-Lafon. An algorithm for distributed groupware applications. In Proc. of the 13th International Conference on Distributed Computing Systems, May 1993, pp.195--202.

[9] M. Knister and A. Prakash. Issues in the design of a toolkit for supporting multiple group editors. In The Journal of the Usenix Association, 6 (2), 1993, pp.135--166.

[10] L. J. McGuffin and G. M. Olson. ShrEdit: a shared electronic workspace. In CSMIL Technical report \#45, University of Michigan, Ann Arbor, 1992.

[11] T. P. Moran, K. McCall, B. van MelIe, E. Ronby Pedersen, and F.G.H. alasz. Some design principles of sharing in Tivoli, a whiteboard meeting support tool. In S. Greenberg, S. Hayne, and R. Rada (eds.), Groupware for Real-time Drawing: A Designer s guide, McGraw-Hill, 1995, pp.24-36.

[12] J. P. Munson and P. Dewan. A concurrency control framework for collaborative systems. In Proceedings of ACM CSCW'1996, pp. 278--287.

[13] R. E. Newman-Wolfe, et al. MACE: a fine grained concurrent editor. In Proc. of the ACM COCS Conference on Organizational Computing Systems, Nov. 1991, pp.240-254.

[14] D. Nichols, P. Curtis, M. Dixon, and J. Lamping. Highlatency, low-bandwidth windowing in the Jupiter collaboration system. In Proc. of ACM Symposium on User Interface Software and Technologies, Nov. 1995, pp.111-120.

[15] A. Prakash. Group editors. In M. Beaudouin-Lafon (ed.), Computer Supported Cooperative Work, John Wiley \& Sons, 1999, pp.103--133.

[16] A. Prakash, and M. Knister. A framework for undoing actions in collaborative systems. In ACM Transactions on Computer-Human Interaction, 4(1), Dec. 1994, pp.295--330.

[17] M. Ressel, D. Nitshce-Ruhland, and R. Gunzenbaeuser. An integrating, transformation-oriented approach to concurrency control and undo in group editors. In Proceedings of the ACM Conference on Computer Supported Cooperative Work, Nov. 1996, pp.288--297.

[18] C. Sun and C.A. Ellis. Operational transformation in realtime group editors: Issues, algorithms, and achievements. In Proceedings of ACM Conference on Computer Supported Cooperative Work, Nov. 1998, pp.59--68.

[19] C. Sun, X. Jia, Y. Zhang, Y. Yang, and D. Chen. Achieving convergence, causality-preservation, and intentionpreservation in real-time cooperative editing systems. In ACM Transactions on Computer-Human Interaction, 5(1), Mar. 1998, pp.63--108.

[20] C. Sun and R. Sosič. Optional locking integrated with operational transformation in distributed real-time group editors. In Proceedings of ACM 18th Symposium on Principles of Distributed Computing, May 1999, pp.43--52.

[21] L. Xue, K. Zhang, and C. Sun. Conflict control locking in distributed cooperative graphics editing systems. In Proceedings of the $1^{\text {st }}$ international conference on Web Information Systems Engineering (WISE 2000), Hong Kong, IEEE CS Press, June 2000, pp.401--408. 